\title{
Comparison of Live Person Test to Head and Hand Phantom Test in Reverberation Chamber
}

\author{
John Kvarnstrand ${ }^{1}$, Susanne Schilliger Kildal ${ }^{1}$, Anton Skårbratt ${ }^{1}$, Madeleine Schilliger Kildal ${ }^{1}$ \\ ${ }^{1}$ Bluetest AB, Göteborg, Sweden, <firstname.lastname@bluetest.se>
}

\begin{abstract}
The Total Radiated Power of a cell phone was measured in a reverberation chamber with a head and hand phantom and compared to live person testing of 5 test subjects who held device in similar grip as the phantom. Repeatability in tests was examined for the phantom and for the live person test subjects. The method demonstrated can be used to evaluate mobile devices handled by live persons. It can also be used to validate that a phantom is a good representation of live persons.
\end{abstract}

Index Terms-Reverberation Chamber, Total Radiated Power, Mobile Device, Head and Hand Phantom

\section{INTRODUCTION}

The number of wireless devices that are either hand held or body worn is steadily increasing. It is becoming more and more important to characterize how well devices are working in the close presence of a person.

At present, the most common method to include the effect of the user in measurements has been to use a head and hand phantom. The head and hand phantoms are designed to simulate the geometry and electromagnetic properties of typical users. Phantoms have many benefits for testing, they are repeatable and can be produced in many identical units, which facilitate reproducible tests between different labs. However, they also have shortcomings. It is difficult to cover all the various ways that a real life user can chose to handle a wireless device. There is also a large spread in the anatomy of users, which is hard to capture with a single phantom.

Performing measurements with real users addresses these issues. Live person testing in a reverberation chamber has become a useful tool for evaluating the influence of the person on the radiation performance of wireless devices that are either hand held [1] or body-worn [2].

The reverberation chamber offers many benefits compared to the traditional antenna pattern test for live person testing. For example the user can move around in a reverberation chamber, compared to the requirement of being perfectly still in an antenna pattern test.

This paper examines how repeatable and representative live person measurements are compared to measurements with a hand and head phantom.

\section{MEASUREMENT SET-UP}

Comparisons between the live person and head and hand phantom, were realized through reference measurements and total radiated power (TRP) measurements. The reference measurements were done by using a vector network analyzer and for the TRP measurements a base station simulator was used. The measurements were also repeated to evaluate the repeatability.

The measurements were performed in a Bluetest RTS90 reverberation chamber (dimensions $3.3 \mathrm{~m} \times 2.55 \mathrm{~m} \times 4.2 \mathrm{~m}$ ). The chamber consist of a large rectangular metal cavity with reflecting walls, mode stirring plates, a fixed multiport antenna used to couple to the resonant modes of the chamber, and a turntable to hold a device under test (DUT) [5][6]. The DUT was a commercially available smart phone, Samsung Galaxy S GT-I9000.

To make the chamber a suitable environment for a person to enter into, it has a door with a handle on the inside, a clear area large enough to move about in, ventilation and light fixtures to illuminate the space. The control software of the measurement system has a self-timer feature that delays the start of the measurement a short time to allow the test operator to enter the chamber and be test subject her/himself if desired.

In the test campaign a head and hand phantom from IndexSAR [3] was used. The phantom was placed on the turntable and the smartphone was mounted in the hand and held toward the face of the phantom according to specification set by CTIA [4], see Figure 1. A reference measurement was performed to get the transmission function of the chamber. Six repeated measurements were performed with the phantom. Between the first 3 configurations the smartphone was removed and then remounted in the grip. Configurations 4-6 were repeated without any modification to the mounting.

For live person test subjects, five volunteering people employed by Bluetest participated, two women and three men of different body size. The people were instructed to hold the phone in a grip as similar as possible to the grip used by the head and hand phantom. They were further instructed to walk back and forth along a U-shaped path inside the chamber, see Figure 2. Each test subject repeated the test 5 to 6 times to determine how repeatable the tests are.

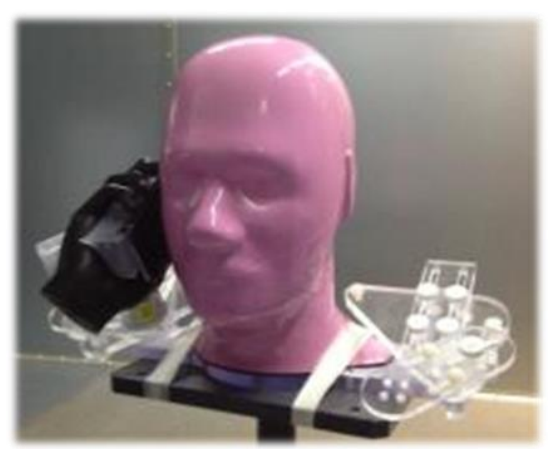

Figure 1. Head and hand phantom used in the test. 


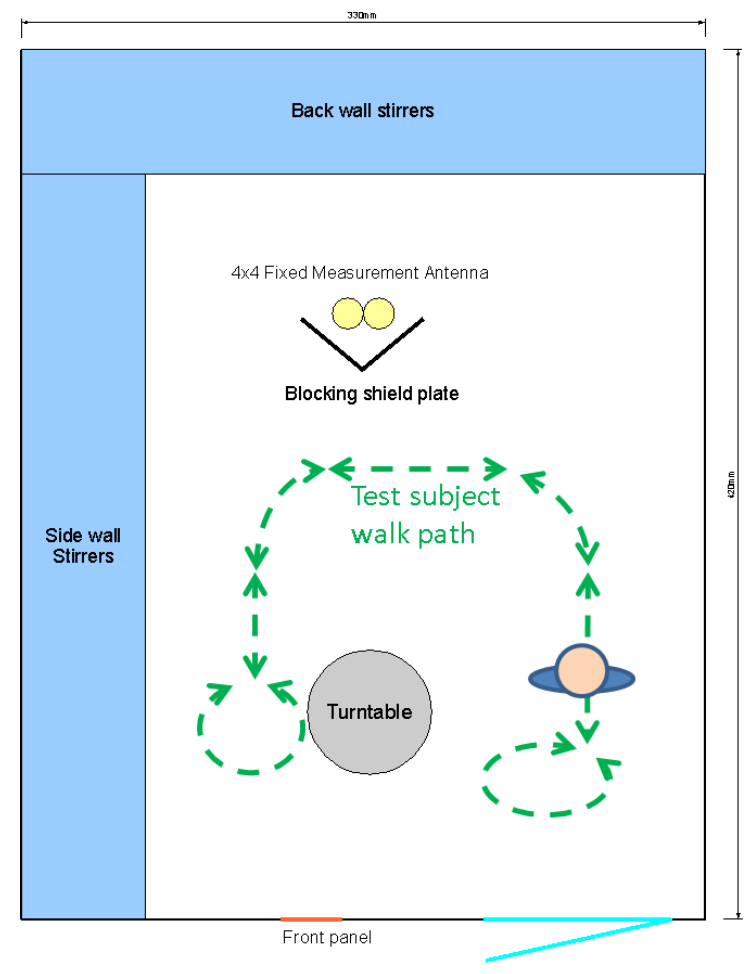

Figure 2. Walk path inside chamber for test subjects.

\section{LOADING EFFECT OF PERSON}

A live person or a phantom in the reverberation chamber adds dissipative loading to the chamber. The losses induced by the person/phantom can be divided into two types, see Figure 3:

A) Direct coupling between device and person/phantom: RF energy emanating from the device is directly absorbed in the person/phantom. A person/phantom in the reactive nearfield of the device can detune the antenna and thereby reduce the radiated power. These effects are strong in the close vicinity of the device.

B) Coupling via one or several reflections in chamber: This effect is present in the reverberation chamber only. It is independent of the distance between the device and phantom.

Effect $\mathrm{A}$ is present in normal use of the device and is therefore desirable to capture in the measurement. Effect B occurs only in the reverberation chamber, which makes it undesired. However, it is easy to remove effect B from the test results by including the person/phantom in the reference measurement.
In this measurement campaign we made a separate reference measurement for each person that was participating and for the phantom. The amount of loading (B) a person induces on the chamber varies between people and depends on the body size. However for repeated test with the same individual the loading effect is very stable even though they make no special effort to move about in the chamber in the same way [1].

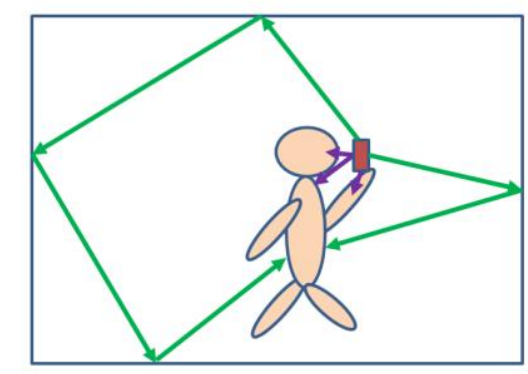
A : Loss from direct coupling between device and person
B : Loss from coupling via one or several reflections in chamber

Figure 3 Loss coupling mechanisms.

\section{MEASUREMENTS}

\section{A. Reference Measurements}

To establish the average transmission loss for the chamber, including the presence of a person or a phantom, reference measurements were made. The reference measurements were performed with a vector network analyzer and a reference antenna with known efficiency. The reference antenna was mounted on the turntable. The transmission loss is measured over a continuous sequence of mode stirring states and the average power is computed. For the phantom case the phantom is placed on the turntable. For the person case each test subject was instructed to walk back and forth along the indicated path, see Figure 2, during the reference measurement.

\section{B. Measurements of Phantom and Several People}

In these experiment we compared the TRP performance of a smartphone with a head and hand phantom and with several real people. Measurements were performed on WCDMA channel 9750 which is at $1950 \mathrm{MHz}$.

The smartphone was mounted in the hand and held toward the face of the phantom according to specification set by CTIA [4]. The phantom was secured to the turntable. A reference measurement was performed to get the transmission function of the chamber. Six repeated measurements were performed with the phantom. Between the first 3 configurations the smartphone was removed and then remounted in the grip. Configuration 46 were repeated without any modification to the mounting. 
After the phantom measurement was completed, tests with live persons were performed. First test subject was person $\mathrm{S}$ who was both test operator and test subject during the test campaign. This was accomplished by using the self-timer feature of the system. Then followed test with person J, C, A and $\mathrm{M}$. In these tests person $\mathrm{S}$ was test operator remaining outside the chamber.

\section{RESUlTS}

\section{A. Reference Measurements}

The results of the reference measurements with live persons were post processed and the results are shown in Figure 4. For person $\mathrm{S}, \mathrm{J}$ and $\mathrm{C}$ the reference measurements were repeated. We find that the spread in loading between different persons is reasonably small, in our study it is about $0.8 \mathrm{~dB}$. Repeated tests with the same person spread at most $0.1 \mathrm{~dB}$. The size of the persons that participated is indicated by their height and weight given in Table I.

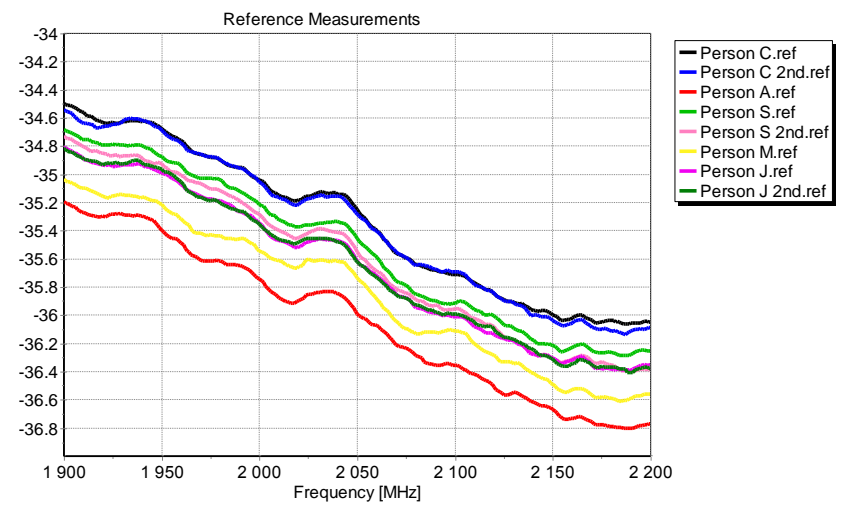

Figure 4. Reference measurements with persons in chamber.

TABLE I.

\begin{tabular}{|c|c|c|c|}
\hline \multicolumn{4}{|c|}{ Height and Weight of Test Subjects } \\
\hline Test Subject & Gender & $\begin{array}{c}\text { Height } \\
(\text { cm })\end{array}$ & $\begin{array}{c}\text { Weight } \\
(\mathbf{k g})\end{array}$ \\
\hline Person C & Female & 165 & 56 \\
\hline Person S & Female & 176 & 60 \\
\hline Person J & Male & 182 & 92 \\
\hline Person M & Male & 189 & 85 \\
\hline Person A & Male & 190 & 85 \\
\hline
\end{tabular}

\section{B. TRP measurement}

The results of the TRP measurements are shown in Figure 5.

The measurements with the phantom repeat very well, even for the first three measurements where the cell phone was removed and reinserted in the hand between measurements.

For the measurements conducted with people, we can see that there is a larger spread between persons than when the same person repeats the same test. Person $\mathrm{S}$ was both test operator and test subject. This meant that the person had to put down the phone to operate the computer between tests and that may explain why these measurements were less repeatable than the other test subjects who could maintain the same grip between the tests.

The variation between results from different people can be attributed to differences in how they hold the phone. Test subjects were instructed to imitate the grip of the phantom as close as possible, but there are limits to how faithfully a person can do that. With regard to the influence of hand size it can be observed that the two female test subjects $\mathrm{S}$ and $\mathrm{C}$ caused less loss than the male test subjects. A possible explanation for this is smaller hand sizes.

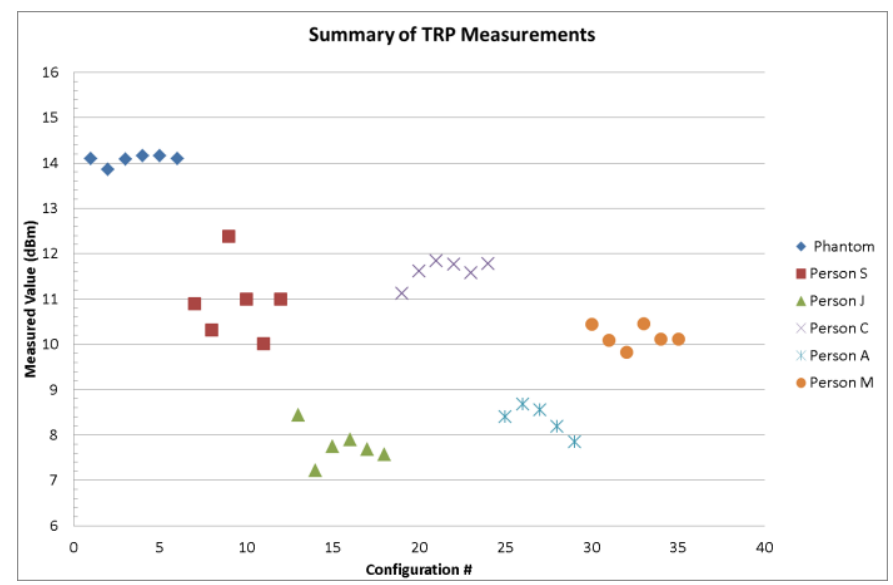

Figure 5. TRP measurements for different persons in the chamber.

\section{CONCLUSIONS AND DISCUSSION}

We have shown how live person tests compare with phantom testing in a reverberation chamber. Tests performed with a phantom are very repeatable and demonstrates the value of phantoms when the same test needs to be repeated with high accuracy. Live person tests are less repeatable, but still remarkably repeatable given that people are not repeatable precision devices. We find that the spread between different people is larger than between repeated measurements by the same person.

In this study we made reference measurements for each person. A clear trend is visible that larger persons load the chamber more than smaller persons. When the same person is measured twice the loading effect repeats very well. However, the loading effect difference between people varied not more than $0.8 \mathrm{~dB}$ in our study. This point to an opportunity to use a single reference measurement with a medium sized person and use it for several people, on the condition that the added uncertainty is acceptable. This could save time and simplify the measurements when it is desired to employ many different test subjects.

The method demonstrated herein can be used to evaluate mobile devices handled by live persons. It can also be used to validate that a phantom is a good representation of live persons. 


\section{REFERENCES}

[1] John Kvarnstrand, Robert Rehammar, Anton Skårbratt, Christian Lötbäck Patané, “A Practical Method to Measure Total Radiated Power of a Mobile Device Handled by a Live Person", Antennas and Propagation, 2013. EuCAP 2013.

[2] Gareth A. Conway, William G. Scanlon, Charlie Orlenius, and Chris Walker, "In Situ Measurement of UHF Wearable Antenna Radiation Efficiency Using a Reverberation Chamber", IEEE Antennas and Wireless Propagation Letters, Vol. 7, 2008

[3] IndexSAR, "Phantoms and fixtures for OTA (Over The Air) wireless testing according to CTIA Test Plan Rev. 3.1”, January 2011
[4] CTIA, "CTIA certification test plan for mobile station over the air performance," Method Meas. Radiated RF Power and Receiver performance, Apr. 2005

[5] M. Andersson, A. Wolfgang, C. Orlenius, J. Carlsson, "Measuring Performance of 3GPP LTE Terminals and Small Base Stations in Reverberation Chambers", Chapter 12 of "Long Term Evolution: 3GPP LTE Radio and Cellular Technology", CRC Press, 2009.

[6] P.-S. Kildal, X. Chen, C. Orlenius, M. Franzén, C. Lötbäck Patané, "Characterization of Reverberation Chambers for OTA Measurements of Wireless Devices: Physical Formulations of Channel Matrix and New Uncertainty Formula," IEEE Transactions on Antennas and Propagation, Vol. 60, No. 8, pp. 3875-3891, Aug. 2012. 